\title{
Estimating uncertainties in hydraulically- modelled rating curves for discharge time series assessment
}

\author{
Valentin Mansanarez ${ }^{1,2, *}$, Ida K. Westerberg ${ }^{3}$, Steve W. Lyon ${ }^{1,2,4}$, and Norris Lam $^{1,2}$ \\ ${ }^{1}$ Stockholm University, Department of Physical Geography, Stockholm, Sweden \\ ${ }^{2}$ Bolin Centre for Climate Research, Stockholm University, Stockholm, Sweden \\ ${ }^{3}$ IVL Swedish Environmental Research Institute, Stockholm, Sweden \\ ${ }^{4}$ The Nature Conservancy, New Jersey Office, New Jersey, USA
}

\begin{abstract}
Establishing a reliable stage-discharge (SD) rating curve for calculating discharge at a hydrological gauging station normally takes years of data collection. Estimation of high flows is particularly difficult as they occur rarely and are often difficult to gauge in practice. At a minimum, hydraulicallymodelled rating curves could be derived with as few as two concurrent SD and water-surface slope measurements at different flow conditions. This means that a reliable rating curve can, potentially, be developed much faster via hydraulic modelling than using a traditional rating curve approach based on numerous stage-discharge gaugings. In this study, we use an uncertainty framework based on Bayesian inference and hydraulic modelling for developing SD rating curves and estimating their uncertainties. The framework incorporates information from both the hydraulic configuration (bed slope, roughness, vegetation) using hydraulic modelling and the information available in the SD observation data (gaugings). Discharge time series are estimated by propagating stage records through the posterior rating curve results. Here we apply this novel framework to a Swedish hydrometric station, accounting for uncertainties in the gaugings and the parameters of the hydraulic model. The aim of this study was to assess the impact of using only three gaugings for calibrating the hydraulic model on resultant uncertainty estimations within our framework. The results were compared to prior knowledge, discharge measurements and official discharge estimations and showed the potential of hydraulically-modelled rating curves for assessing uncertainty at high and medium flows, while uncertainty at low flows remained high. Uncertainty results estimated using only three gaugings for the studied site were smaller than $\pm 15 \%$ for medium and high flows and reduced the prior uncertainty by a factor of ten on average and were estimated with only 3 gaugings.
\end{abstract}

\section{Introduction}

Despite the fundamental importance of discharge time series for hydrology, discharge time series are rarely measured directly. Instead, stage-discharge (SD) rating-curve methods are traditionally used to empirically derive discharge time series from stage records. Rating curves

\footnotetext{
*e-mail: valentin.mansanarez@natgeo.su.se
} 
typically consist of single or multi-section power-law functions calibrated using numerous gaugings, i.e. one-off SD measurements $[1,2]$. These traditional methods are typically associated with several sources of uncertainty that have led to the development of various rating-curve uncertainty estimation methods [e.g., 3-8].

A main challenge with traditional methods is that it can take many years of data collection efforts to establish a reliable SD rating curve at a hydrometric station. This is because it is practically difficult to take gaugings that cover the whole variability of the SD relation, particularly in small catchments with a fast rainffall-runoff response [9]. In particular, traditional rating curves are often uncertain for high flows since they occur rarely (giving few opportunities to gauge them), and they are often practically difficult to gauge. This means that the highest flows typically need to be extrapolated, often using some hydraulic considerations of the high-flow SD relation.

Hydraulic modelling has proven to be a good alternative for estimating rating curves, in particular for more reliable high flow discharge estimation $[10,11]$. This is because hydraulic models can exploit information about the physical configuration of the channel [e. g., from airborne laser scans, 12] thereby deriving rating curves using only a few gaugings. This allows the SD relation to be derived much faster than with traditional methods. However, hydraulically-modelled rating curves are still affected by the combined uncertainty in the model parameterisation, the gauging data and any temporal changes in the SD relation [11].

In this paper, we use a novel uncertainty framework built on Bayesian inference and a physically-based hydraulic rating curve model. This framework can be used for developing SD rating curves and estimating their uncertainties at hydrometric stations. The onedimensional hydraulic rating curve model from Kean and Smith [13] forms the hydraulic model core of the framework. Here, we apply this uncertainty framework to a Swedish site as a case study. As such, the aim of this study is to assess the impact of using only three gaugings for calibrating a hydraulic model (with explicit prior distributions) on the resultant posterior uncertainty estimations within our framework. The rating curve uncertainty is then propagated to discharge time series to assess its implication for discharge time series data.

\section{Method}

\subsection{Hydraulically modelled rating curves}

The simplified one-dimensional hydraulic model from Kean and Smith [13, 14] is used to derive a rating curve. This model has been developed for relatively straight reaches with a ratio of length to width of at least 7 [13]. For computing flow, the model uses the river shape (main channel plus floodplain), which is usually obtained through topography surveys.

In the hydraulic model, the resistance to the flow is defined as a roughness height, $z_{0}$. This physical variable, which corresponds to the roughness of the channel bank and the streambed material, is modelled as an average value for the whole stream reach. The model can, however, incorporate different roughness characteristics along the stream reach, such as the location and roughness values of vegetation on the banks or the presence of specific small roughness elements within the channel. These roughness characteristics can be quantified through geometric measurements such as pebble count surveys or vegetation density surveys [12]. At location where these roughness characteristics are provided, the roughness height parameter, $z_{0}$, no longer applies.

Contrary to the previous versions of the hydraulic model [12-14], in this paper, the roughness height, $z_{0}$, is not fixed manually or through back calculation [14]. Instead it is modelled as an independent parameter in the uncertainty framework. This is advantageous as it allows specifying the uncertainty of this parameter. 
Similar to Lam et al. [12], we model the water surface slope, $S$, as a linear function of stage, $h$ :

$$
S=\frac{S_{2}-S_{1}}{h_{2}-h_{1}} h+\frac{S_{1} h_{2}-S_{2} h_{1}}{h_{2}-h_{1}}
$$

where $S_{1}$ and $S_{2}$ are two distinct average water slope values for two distinct stages $h_{1}$ and $h_{2}$ respectively. Please note that eq. (1) means that at least two distinct slope values $S_{1}$ and $S_{2}$ are needed to compute the water slope-stage relation in this version of the hydraulic model.

Once the geometry of the studied river and the water slope-stage relation have been set up, the modelling procedure consists of computing velocity profiles, using the water slopestage relation (from eq. (1)), for every submerged point of the river grid in the modelling domain. This is done by numerically solving the one-dimensional flow equations for steady non-uniform flow for a given stage value. The rating curve is then derived by repeating this modelling procedure for different values of stage.

A full model description and example applications, without estimation of model uncertainty, are available from several previous studies [e.g., 12-17].

\subsection{Uncertainty framework}

Uncertainties in the hydraulically-modelled rating curves are assessed with Bayesian inference [18]. The following section describes the uncertainty framework considered here and we refer to Gelman et al. [18] for a full description of the Bayesian inference.

To begin, let $\boldsymbol{\theta}=\left(\theta_{1}, \theta_{2}, \theta_{3}\right)$ denote the inferred model parameters. These parameters correspond, in this order, to the following parameters of the hydraulic model (see section 2.1): the roughness height $z_{0}$ and the two water slope values, $S_{1}$ and $S_{2}$, used in the water surface slope model (eq. (1)).

The gaugings $\left(\breve{h}_{i}, \widetilde{Q}_{i}\right)_{i=1, N}$ are seen as $N$ estimates of the real values $\left(h_{i}, Q_{i}\right)_{i \in 1, N}$ of stages and associated discharges. We assume that stage errors are negligible compared to discharge errors:

$$
\left\{\begin{array}{l}
\widetilde{h}_{i}=h_{i} \\
\widetilde{Q}_{i}=Q_{i}+\epsilon_{Q, i} \quad \text { with } \quad \epsilon_{Q, i} \stackrel{\text { indep. }}{\sim} \mathcal{N}\left(0, u_{Q, i}\right)
\end{array}\right.
$$

where the discharge errors $\epsilon_{Q}=\left(\epsilon_{Q, 1}, \ldots, \epsilon_{Q, N}\right)$ are assumed independent (indep.) and the standard deviations $u_{Q, i}$ (i.e. the uncertainties of the discharge measurements) are assumed to be known. If no specific information about measurement uncertainties is provided with the gaugings, uncertainty values can be assigned based on typical literature values from measurement error analyses [e.g., 19], or in-situ intercomparisons [e.g., 20].

The true discharge is then written as the discharge predicted by the hydraulic model $f$ :

$$
Q_{i}=f\left(h_{i} \mid \boldsymbol{\theta}\right)
$$

Combining eqs. (2) and (3) yields the following stage-discharge (SD) relation between observed values:

$$
\widetilde{Q}_{i}=f\left(\widetilde{h}_{i} \mid \boldsymbol{\theta}\right)+\epsilon_{Q, i} \quad \text { with } \quad \epsilon_{Q, i} \stackrel{\text { indep. }}{\sim} \mathcal{N}\left(0, u_{Q, i}\right)
$$

Note that in eqs. (3) and (4), contrary to e.g. the Baratin framework [5], we only consider the parametric uncertainty. This is partly because we use too few gaugings to be able to estimate the parameters of a statistical error model.

Equation (4) allows computing the following likelihood function $L$ :

$$
L(\widetilde{\boldsymbol{Q}} \mid \boldsymbol{\theta}, \tilde{\boldsymbol{h}})=\prod_{i=1}^{N} p_{\text {norm }}\left[\widetilde{Q}_{i} \mid f\left(\widetilde{h}_{i} \mid \boldsymbol{\theta}\right), u_{Q, i}\right]
$$


where $\widetilde{\boldsymbol{Q}}=\left(\widetilde{Q}_{1}, \ldots, \widetilde{Q}_{N}\right)$ are the $N$ gauged discharges, $\widetilde{\boldsymbol{h}}=\left(\widetilde{h}_{1}, \ldots, \widetilde{h}_{N}\right)$ are the $N$ observed stage values and $p_{\text {norm }}[z \mid m, s]$ denote the probability density function of a Gaussian distribution with mean $m$ and standard deviation $s$, evaluated at some value $z$. The likelihood models the information available in the observed data.

In addition to the information obtained from the observed data (i.e. the gaugings), Bayesian inference allows us to include any other relevant information - independent from the gaugings - in the form of prior distributions. The specification of priors is site-specific and those used in this study are described in section 3.2.

The likelihood combined with the prior distribution for $\boldsymbol{\theta}$ yield the posterior distribution. This distribution is then estimated using Markov Chain Monte Carlo (MCMC) sampling where each sample gives possible values of the parameters of the hydraulic model, i.e a possible rating curve. In this study, 4 MCMC chains of 15,000 simulations each were drawn to evaluate the algorithm convergence with the Gelman-Rubin criterion [21]. Each chain was burned with a factor of $1 / 3$, i.e. the 5,000 first simulations were discarded. The 10,000 remaining simulations of each chain were slimmed by a factor of 10 before further use. This reduction (slimming) gave a total number of 4,000 MCMC samples in our study. This reduction is made to reduce computing time and storage issues. Further, the reduction did not result in a noticeable loss of information because the raw MCMC are strongly autocorrelated.

Rating curve uncertainty intervals were computed from the MCMC results as well as the maximum a posteriori (MAP) estimator. In this paper, and as usually done in hydrometry applications [22], we only show the uncertainty intervals with a probability level of $95 \%$ ( $2.5 \%$ and $97.5 \%$ percentiles).

\section{Application}

This section presents the application of the uncertainty framework to a case study. First the Swedish study site is described, followed by the prior specification and the posterior rating curve and discharge time series results. In the application, only three gaugings with their associated uncertainty were used to calibrate the model to estimate the rating curve and the related uncertainty. The difference between the prior and posterior uncertainty shows the impact of considering only a few gaugings on subsequent rating curve uncertainty results.

\subsection{The Voxnan River at Nybro, Sweden}

At the Nybro station, the Voxnan River drains a catchment area of $2251 \mathrm{~km}^{2}$ with a mean annual discharge of $25.6 \mathrm{~m}^{3} \cdot \mathrm{s}^{-1}$. The hydrometric station is managed by the Swedish Meteorological and Hydrological Institute (SMHI) and is located just downstream of a motor vehicle bridge (GPS location: 61 $21^{\prime} 46.3^{\prime \prime} \mathrm{N}, 15^{\circ} 31^{\prime} 30.9^{\prime \prime} \mathrm{E}$ ).

During winter, the station is affected by variable backwater due to ice jam downstream. This backwater influence is not considered by the SMHI in the rating curve calibration. For the stable period, the official rating curve has been calibrated by the SMHI using 13 gaugings and it is considered valid since 1991. Two other discharge measurements at medium flow were performed by the SMHI in November 2014 and April 2016 and agree with this calibration. Since this calibration, no more high flow gaugings have been made.

In addition to these 15 available SHMI gaugings, we performed two independent gaugings in October 2013 and May 2016, respectively. These two gaugings, as well as the gauging performed by the SMHI in April 2016 were used as calibration data in this study. It should be noted that in our independent October 2013 measurement variable backwater was observed near the motor vehicle bridge. Therefore, the discharge measurement was taken upstream 
of the stream reach (300 $\mathrm{m}$ from the bridge) to remove the backwater influence. The three discharge measurements, used for calibrating the model in this study, were performed in good conditions using acoustic Doppler current profilers (ADCP). According to the literature [e.g., 23], discharge measurement uncertainty was set to $\pm 5 \%$ for these three gaugings.

Per the model design and setup, we also made three water surface slope surveys in connection with the three discharge observations used for calibration. Two of these were acquired at the same time as our two independent gaugings on October 2013 and May 2016. The third slope survey did not correspond with any of the gaugings performed by the SMHI; however, the survey covered the same flow conditions as observed on the SMHI's April 2016 measurement. Since no major channel changes have been recorded since 1991, the water surface slope survey we made in April 2014 was thus associated to the gauging performed in April 2016 by the SMHI.

The topographic survey of the modelled stream reach was performed on a fairly straight $290 \mathrm{~m}$ long and $30 \mathrm{~m}$ wide reach (ratio of length to width of 9.7). This reach was just upstream of the bridge and covered the reference cross-section where our two independent gaugings were made. From this topography survey, 92 cross-sections were used to model the river shape (one cross-section every $3.2 \mathrm{~m}$ ) and each cross-section had 251 points (spacing of $12 \mathrm{~cm}$ ). The topography survey used in this study did not cover the SMHI staff gauge (located just downstream the motor vehicle bridge). Therefore, a stage offset of $0.8 \mathrm{~m}$ was used to relate the stage data recorded by the SMHI with our hydraulically-modelled rating curve results. This offset was based on staff gauge observations between the SMHI's gauge and a temporary gauge that we installed during the topography survey. No uncertainty in this offset was considered in this study.

Two vegetation (stem density) surveys were also performed along the right bank to measure the roughness due to vegetation. These surveys were taken into account in the hydraulic model as fixed values.

More information on the Nybro station within the Voxnan River is available in Lam [24].

\subsection{Prior specification}

The information available about the station allowed us to specify the prior distributions for the hydraulic model parameters (Table 1). Roughness was not specifically surveyed in the whole stream reach at Nybro site as the roughness height $z_{0}$ was back computed from a discharge value and water slope measurements in the previous versions of the hydraulic model. Therefore, an imprecise uniform distribution $\mathcal{U}(0 ; 0.3)$ was set up as prior distribution on parameter $z_{0}$. For the slope parameters $S_{1}$ and $S_{2}$, the following considerations were used to set up the prior distributions. As variable backwater was observed during the measurements of October 2013, that water surface slope survey was not used to set up the slope parameter priors. Two simple linear regressions were made using the two remaining surveys (May 2016 and April 2014) for parameters $S_{1}$ and $S_{2}$. Normal distributions were taken as priors for these two slope parameters with means and standard deviations estimated from these two regressions. Because the water surface slope measurements of April 2014 were not performed at the same time as the discharge measurement, the uncertainty of this parameter is set to $\pm 100 \%$ to reflect a low confidence.

\subsection{Rating curve results}

The posterior uncertainty intervals were smaller than the prior intervals (by a factor of approximatively ten on average, see fig. 1). The parameter $z_{0}$ was well identified from the three calibration data (see table 1 and fig. 2). The $z_{0}$ posterior distribution was more precise than 
Table 1. Parameters, prior distributions and posterior results for the application of the uncertainty framework to the Nybro site. The symbol $\mathcal{N}(\mu, \sigma)$ corresponds to the normal distribution with mean $\mu$ and standard deviation $\sigma$. The symbol $\mathcal{U}(a, b)$ corresponds to the uniform distribution on the interval $[a, b]$.

\begin{tabular}{ccc}
\hline $\begin{array}{c}\text { Physical } \\
\text { parameter }\end{array}$ & Prior & $\begin{array}{c}\text { Posterior results: } \\
\text { MAP [2.5\% and 97.5\% percentiles] }\end{array}$ \\
\hline $\begin{array}{c}z_{0}[\mathrm{~m}] \\
S_{1}[\mathrm{~m} / \mathrm{m}]\end{array}$ & $\mathcal{N}\left(-3.409 \times 10^{-5} ; 1.273 \times 10^{-5}\right)$ & $-3.478 \times 10^{-5}\left[-6.779 \times 10^{-5} ;-2.711 \times 10^{-5}\right]$ \\
$S_{2}[\mathrm{~m} / \mathrm{m}]$ & $\mathcal{N}\left(-1.247 \times 10^{-4} ; 6.237 \times 10^{-5}\right)$ & $-6.571 \times 10^{-5}\left[-1.207 \times 10^{-4} ;-4.820 \times 10^{-5}\right]$ \\
\hline
\end{tabular}

its prior distribution and agrees with parameter values found by Lam [24]. The second slope parameter $S_{2}$ is also (albeit less) identified from the calibration data. The $S_{2}$ posterior distribution is no longer based on the prior but still remains uncertain. The first slope parameter $S_{1}$ remained similar to the prior distribution.

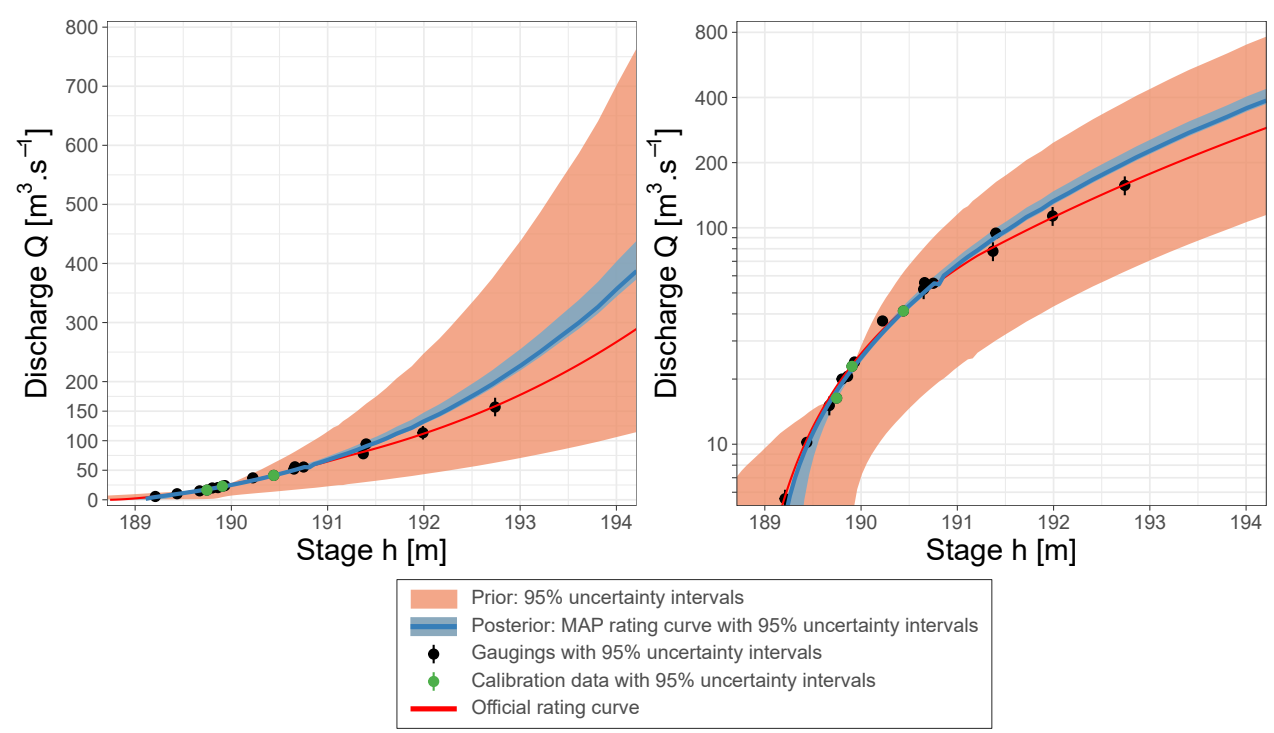

Figure 1. The Voxnan River at Nybro, Sweden: stage-discharge representation of the results from the uncertainty framework using hydraulic rating-curve modelling.

With only three observations as calibration data, rating curve uncertainty was clearly reduced compared to the prior for medium and high flows, but remained high for low flows (Figure 1). At medium flows, the MAP rating curve was in good agreement with the gaugings (discharge error of less than $\pm 10 \%$ ) and the associated uncertainty intervals were within $\pm 10 \%$. At higher flows, the uncertainty intervals were within $\pm 15 \%$ for the extrapolation beyond the highest measured discharge $\left(157 \mathrm{~m}^{3} \cdot \mathrm{s}^{-1}\right.$ for a stage value of $\left.192.74 \mathrm{~m}\right)$. At the highest observed stage $(193.5 \mathrm{~m})$, the discharge uncertainty was relatively constrained $(-4 \%$ and $+13 \%$ ). However, the estimated MAP rating curve no longer followed the gaugings. It should be noted that the two highest high-flow gaugings were taken in May 1995. There is some reason to suspect that the disagreement between the MAP rating curve and these two high-flow gaugings could be explained by a change in the stage-discharge (SD) relation. This is supported by the fact that the MAP rating curve passes close to a more recent high-flow 


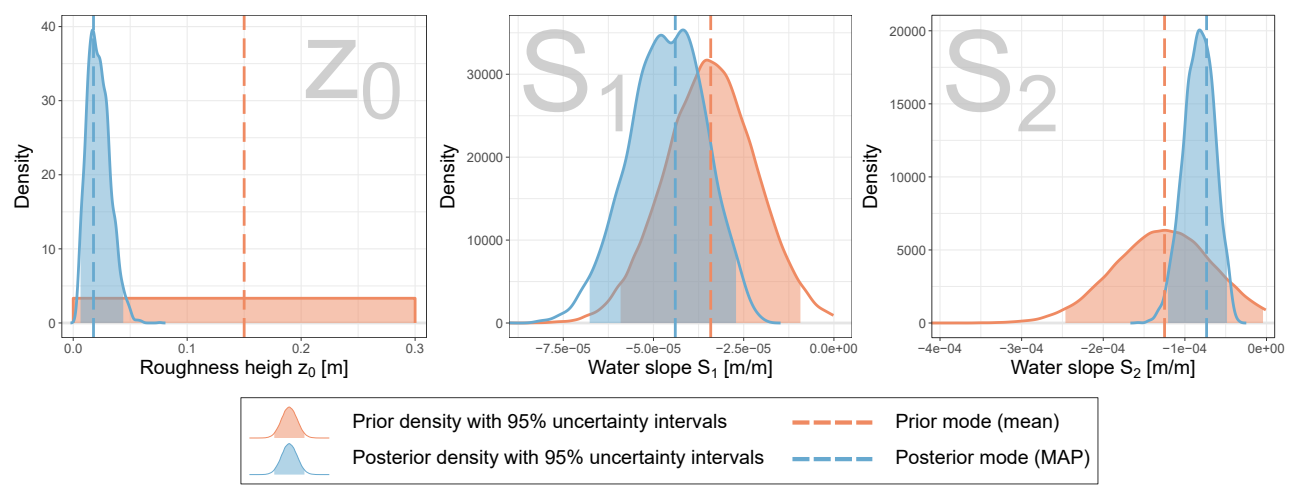

Figure 2. The Voxnan River at Nybro, Sweden: prior and posterior densities of the parameter results from the uncertainty framework using hydraulic rating-curve modelling.

gauging, taken in 2008. However, without other additional recent gaugings, it is impossible to confirm if there have been a rating change or not in the SD relation.

The lower flows yield questionable posterior results and the rating curve results are uncertain. The MAP rating curve underestimates discharges (up to $-20 \%$ for low flows) and relative uncertainty intervals are large (up to $+170 \%$ for the very lowest flows). Several causes could explain this: (1) more prior information and/or observations may be required or (2) a more precise topography may lead to better low-flow results. This poor performance at low flows could also be explained either by the fact the model is not well adapted to low-flow conditions. Specifically, the model assumptions (i.e. a ratio of length to width of at least 7) may begin to be violated for flows which are more unsteady or by some physical change in the SD rating curve. Notedly for the latter condition, the lowest gauging one record was performed in 1992 before the high flood event of 1995. Furthermore, the spacing of the SD gaugings across the flow range will affect the resultant rating-curve uncertainty. Future analyses will be made to investigate at what flow conditions measurements should optimally be taken to reduce uncertainty.

\subsection{Discharge time series}

Figure 3 represents the resulting uncertain discharge time series at Nybro for the time period of April to June 2016. Two of the gaugings used in the calibration process were performed during this period, which correspond to low and medium flows. The stage records of this period were propagated into discharge time series using the posterior rating curve results from section 3.3. In this propagation, the stage errors were ignored such that the uncertainty intervals only encompass the rating curve errors.

During this time period, the discharge errors between the MAP curve and the official results were less than $\pm 6 \%$. Further, the MAP curve was in agreement with the medium flow gauging (April 2016) and the uncertainty bounds were within $\pm 10 \%$. On other time periods, at very low flows, discharges were underestimated by the model (MAP errors of up to $-20 \%$ ) and discharge estimations were more uncertain (uncertainty bounds of up to $\pm 90 \%$ ).

\section{Conclusions}

A novel Bayesian method using hydraulically-modelled rating curves was applied for estimating stage-discharge rating curves and their related uncertainties. 


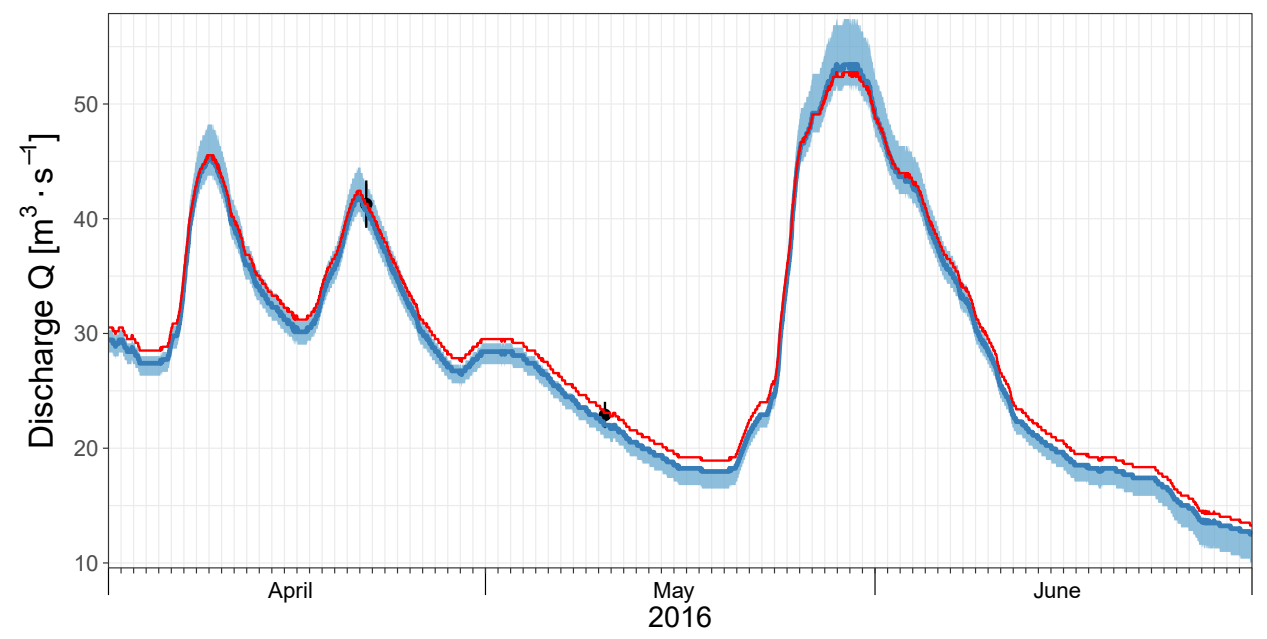

Figure 3. Discharge time series with uncertainty for the Voxnan River at Nybro, Sweden, April to June 2016. The MAP rating curve is shown as a blue line with the related $95 \%$ posterior uncertainty intervals; in red, the official SMHI discharge time series; and in black the gaugings with their $95 \%$ uncertainty intervals.

The main difference to the previous version of the hydraulic modelling procedure is that the roughness height parameter, $z_{0}$ is no longer back computed from water surface slope and discharge measurements. Rather this parameter is modelled as a complete independent parameter for which prior information can be specified. The hydraulic model thus only needs a topographic survey of the hydrometric station but can still incorporate several specific located roughness values on the bank of the river (such as vegetation stem density).

The potential of the new method was illustrated by applying it to a stable stage-discharge relation at a Swedish site. The roughness parameter could be identified with only three gaugings and precise prior information on one of the water slope parameters. The results showed a clear reduction of the prior uncertainty at medium and high flows, where the resulting ratingcurve results showed acceptable agreement with the gaugings and the uncertainty intervals were within $\pm 15 \%$ for the extrapolation at high flows. Low-flow uncertainty estimations remained large, yielding less convincing results. Clearly, more investigation is needed to understand the interplay between model parameters, observational errors and uncertainty impacts within our framework.

From this initial assessment of our uncertainty framework, there are advantages to our approach of being calibrating using only a few gaugings (e.g. three are used here) and that it is not the case that these gaugings must contains high-flow discharge measurements. It therefore allows establishment of a rating curve much faster than traditional rating curve methods. Uncertainty results from this method come as sampled probabilistic parameter distributions, thereby enabling the uncertainty to be propagated to discharge time series and any relevant statistics (e.g., hydrological signature values).

\section{Acknowledgement}

The financial support of The Swedish Research Council Formas (Svenska Forskningsrådet Formas) [942-2015-321] is gratefully acknowledged. The authors also acknowledge the Pro- 
fessor Dmitri Kavetski (University of Adelaide, Australia) for the use of his numerical Fortran library (DMSL). We thank the SMHI (Swedish Meteorological and Hydrological Institute) for sharing the gauging datasets and the stage records of the Nybro station. Finally, we would like to thank the two anonymous reviewers for their time and efforts.

\section{References}

[1] S. E. Rantz, Measurement and computation of streamflow. Volume 2: Computation of discharge (Water-Supply Paper 2175, 1982), 631 p.

[2] A.R. Schmidt, Analysis of stage-discharge relations for open-channel flows and their associated uncertainties, $\mathrm{PhD}$ dissertation (2002)

[3] T. Reitan, A. Petersen-Øverleir, Stochastic Environmental Research and Risk Assessment, 23, 16 p. (2009)

[4] A. E. Sikorska, A. Scheidegger, K. Banasik, J. Rieckermann, Hydrol. Earth Syst. Sci., 17, 13 p. (2013)

[5] J. Le Coz, B. Renard, L. Bonnifait, F. Branger, R. Le Boursicaud, J. Hydrol., 509, 573587 (2014)

[6] T. Morlot, C. Perret, A.-C. Favre, J. Jalbert, J. Hydrol., 517, 14 p. (2014)

[7] H. K. McMillan, I. K. Westerberg, Hydrol. Process., 29, 10 p. (2015)

[8] G. Coxon, J. Freer, I. K. Westerberg, T. Wagener, R. Woods, P. J. Smith, Water Resour. Res., 51, 16 p. (2015)

[9] I. K. Westerberg, T. Wagener, G. Coxon, H. K. McMillan, A. Castellarin, A. Montanari, J. Freer, Water Resour. Res., 52, 19 p. (2016)

[10] M. Lang, K. Pobanz, B. Renard, E. Renouf, E. Sauquet, Hydrol. Sciences J., 55, 17 p. (2010)

[11] G. Di Baldassarre, P. Claps, Hydrol. Res., 42, 10 p. (2011)

[12] N. Lam, J. W. Kean, S. W. Lyon, Hydrol. Res., 48, 17p. (2016)

[13] J. W. Kean, J. D. Smith, J. Geophys. Res., 115, 15 p. (2010)

[14] J. W. Kean, J. D. Smith, Kansas, J. Geophys. Res., 110, 17 p. (2005)

[15] J. A. Clayton, J. W. Kean, Water Resour. Manag., 24, 24 p. (2010)

[16] M. Nathanson, J. W. Kean, T. J. Grabs, J. Seibert, H. Laudon, S. W. Lyon, Hydrol. Process., 26, 8 p. (2012)

[17] S. W. Lyon, M. Nathanson, N. Lam, H. E. Dahlke, M. Rutzinger, J. W. Kean, H. Laudon, Water, 7, 16 p. (2015)

[18] A. Gelman, J. B. Carlin, H. S. Stern, D. B. Dunson, A. Vehtari, D. B. Rubin, Bayesian Data Analysis, (Texts in Statistical Science, third ed., Chapman \& Hall., 2014)

[19] A. Despax, C. Perret, R. Garçon, A. Hauet, A. Belleville, J. Le Coz, A.-C. Favre, J. Hydrol., 533, 13 p. (2016)

[20] J. Le Coz, B. Blanquart, K. Pobanz, G. Dramais, G. Pierrefeu, A. Hauet and A. Despax, J. Hydraul. Eng., 142, 15 p. (2016)

[21] A. Gelman, D. B. Rubin, Statistical Sciences, 7,16 p. (1992)

[22] ISO/TS 25377:2007, Hydrometric Uncertainty Guidance, International Organization for Standardization, 51 p. (2007)

[23] K. Lee, H.-C- Ho, M. Muste, C.-H. Wu, J. Hydrol., 509, 14 p. (2014)

[24] N. Lam, Modeling rating curve from close-range remote sensing data. Application of laser and acoustic ranging instruments for capturing stream channel topography, $\mathrm{PhD}$ dissertation (2017) 